De Tampere à Séville : bilan de la sécurité européenne $(1 / 2)$

\title{
Les répercussions du Traité d'Amsterdam et de la Déclaration de Tampere sur les Institutions de Police
}

Repercussions of the Amsterdam Treaty and the Tampere Declaration on Police Institutions

\section{Malcolm Anderson}

Traducteur : Nicolas Wuest-Famôse

\section{OpenEdition Journals}

Édition électronique

URL : http://journals.openedition.org/conflits/781

DOI : $10.4000 /$ conflits. 781

ISSN : $1777-5345$

Éditeur :

CCLS - Centre d'études sur les conflits lilberté et sécurité, L'Harmattan

Édition imprimée

Date de publication : 1 mars 2002

Pagination : 115-123

ISBN : 2-7475-3029-9

ISSN : 1157-996X

\section{Référence électronique}

Malcolm Anderson, «Les répercussions du Traité d'Amsterdam et de la Déclaration de Tampere sur les Institutions de Police », Cultures \& Conflits [En ligne], 45 I printemps 2002, mis en ligne le 22 mars 2006, consulté le 30 mars 2021. URL : http://journals.openedition.org/conflits/781 ; DOI : https:// doi.org/10.4000/conflits.781

Ce document a été généré automatiquement le 30 mars 2021.

Creative Commons License 


\title{
Les répercussions du Traité d'Amsterdam et de la Déclaration de Tampere sur les Institutions de Police
}

\author{
Repercussions of the Amsterdam Treaty and the Tampere Declaration on Police \\ Institutions
}

Malcolm Anderson

Traduction : Nicolas Wuest-Famôse

1 Deux difficultés se présentent pour évaluer ces répercussions ${ }^{1}$. Les premières tiennent au temps de l'analyse : à court terme, les effets d'Amsterdam et de Tampere seront certainement faibles ; à long terme, ils seront probablement diffus et involontaires. Les secondes sont redevables au fait que les institutions, y compris celles de la police, sont notoirement difficiles à définir ou à appréhender notamment parce qu'elles recouvrent des concepts différents, enracinés dans une pluralité de traditions intellectuelles.

2 Cette diversité peut trouver une illustration dans le monde académique - bien que beaucoup de leurs praticiens n'acceptent pas des descriptions sommaires et stéréotypées. Pour les sociologues, les institutions sont des discours et des comportements répétés à partir desquels on peut établir des modèles à vocation explicative. Pour les juristes, les institutions sont créées par des textes juridiques et tiennent leur légitimité de ceux-ci: les institutions sont constituées d'une suite de règles astreignantes et exécutoires. Pour les politologues, les institutions sont faites de ces règles et arrangements incarnés dans, et exprimés par, les autorités publiques. Voilà le problème dès lors qu'il s'agit d'évaluer de telles répercussions en ayant pour grille d'analyse les perspectives de ces différentes disciplines.

3 L'approche sociologique est la plus prometteuse en termes d'impact sur le plan du Traité d'Amsterdam et de la déclaration de Tampere. Mais l'approche sociologique requiert aussi bien d'aller travailler sur le terrain que de mener des recherches 
soigneuses. Pour le sociologue, des difficultés se présentent à vouloir séparer l'influence d'Amsterdam et de Tampere des diverses pressions qui modèlent le comportement et le discours de la police. Quoiqu'il en soit, c'est dans l'attitude et le discours de la police que Tampere et Amsterdam risquent d'être les plus influents, car c'est au niveau symbolique que le Traité - et dans une moindre mesure la Déclaration donne une plus grande légitimité et une plus grande visibilité à diverses formes de coopération transfrontalière des polices, de même qu'il encourage de nouvelles initiatives. Mais il demeure impossible pour le moment de savoir si, et comment, ces opportunités seront saisies.

4 C'est le juriste qui rencontre le plus de difficultés à identifier de telles répercussions parce que le Traité d'Amsterdam et la Déclaration de Tampere sont, à quelques exceptions près, des textes déclaratoires avec une série d'objectifs à atteindre (dans le cas d'Europol, quatre objectifs doivent être atteints dans un délai de cinq ans), plutôt qu'un ensemble de règles juridiques. La réalisation progressive d'un espace de liberté, de sécurité et de justice, couplée à des progrès dans le domaine de la libre circulation des personnes, les contrôles aux frontières extérieures, le droit d'asile, l'immigration, ou encore la protection des droits des ressortissants de pays tiers, de même que la coopération dans les affaires criminelles, auront tous et très certainement un impact juridique à long terme. Il en découlera des modifications sur les institutions policières et sur les juridictions auxquelles elles doivent légalement rendre compte. Mais une analyse juridique complète doit encore attendre les décisions politiques à venir. Même l'adoption d'un mandat d'arrêt européen et la position commune sur le terrorisme ne modifient pas ce constat.

5 C'est donc par défaut que les évaluations préliminaires de l'impact du Traité d'Amsterdam et de la Déclaration d'Amsterdam échoient au politologue. La répercussion est indirecte car les Etats membres conservent la plénitude de leur responsabilité pour faire respecter la loi, l'ordre et le maintien de la sécurité intérieure. Les Etats membres conservent aussi leurs prérogatives dans le domaine de la libre circulation des personnes. Les politiques communes dans les affaires d'immigration et d'asile envisagées par le Traité seront très probablement fondées sur des accords intergouvernementaux et des aménagements nationaux seront sans aucun doute tolérés. L'exécution de ces politiques demeurera sous le contrôle des polices nationales. Le schéma intergouvernemental de cette coopération, dans ce qui s'appelait auparavant «Justice et Affaires intérieures » demeure inchangé. Des adaptations aux circonstances nationales constitueront de fait, un trait essentiel du système en développement d'une police en Europe.

6 Mais ceci ne veut pas dire que rien n'a changé. Du point de vue institutionnel, quatre modifications importantes peuvent être identifiées. Bien que le Conseil des Ministres soit le forum dans lequel la politique en matière de police continue à être décidée, la Commission (après Amsterdam) obtient un rôle d'initiative dans certains domaines. Une nouvelle direction générale pour la liberté, la sécurité et la justice a été créée. Elle est désormais compétente, dans les domaines des activités de police criminelle, pour établir des recherches, une documentation et des données statistiques sur le crime transfrontalier.

7 Ces changements amèneront la Commission à avoir des contacts réguliers avec les représentants des agences de police des Etats membres - même si l'on peut estimer qu'il y aura certainement des tentatives de la part de ces Etats pour canaliser ces 
contacts via les plus hauts fonctionnaires des Ministères de la Justice et de l'Intérieur. Mais l'établissement d'une académie européenne de police et un groupe de travail des chefs de police changeront éventuellement la donne.

8 Les tâches d'Europol dans l'échange d'information et de coordination des enquêtes ont été confirmées dans les domaines médiatiques du racisme, de la xénophobie, du terrorisme, du trafic d'êtres humains et des agressions commises contre les enfants. Il revient aussi à Europol de soutenir une coopération toujours plus étroite entre les autorités judiciaires et d'autres autorités compétentes au sein des Etats membres. La capacité d'Europol d'assumer effectivement tous ces rôles est sévèrement limitée. En particulier à cause de la faiblesse de ses ressources financières et du nombre réduit de son personnel. Sans doute l'effet des événements du 11 septembre 2001 sera de confirmer l'unité anti-terroriste d'Europol comme le lieu privilégié des échanges des renseignements dans ce domaine.

9 Un programme élargi et sans limites fixes a été établi, avec une insistance sur le rôle " opérationnel », sans que l'on sache précisément ce que cela engage. A la lumière de la Convention Europol, du Traité d'Amsterdam et de la déclaration de Tampere, on peut avancer que cette disposition suppose le pouvoir d'initier des enquêtes par une demande aux autorités compétentes des Etats membres, de même que le devoir de soutenir et coordonner les enquêtes lancées par ces mêmes Etats.

Les déclarations fondées sur le Traité apportent un soutien aux ambitions de création d'une véritable agence européenne de police dotée de pouvoirs exécutifs, bien que cela soit spécifiquement exclu par certains Etats membres, comme le Royaume-Uni. Une telle agence est difficilement concevable sans qu'il puisse y avoir une évolution vers des formes plus fédérales de l'Union européenne dans le domaine de la Justice et des Affaires Intérieures. Toutefois, qu'une telle possibilité soit source de discussion (dusset-elle être ultérieurement rejetée) donne une idée de l'importance acquise par Europol et de sa perception par les agences de police des Etats membres. Le fait qu'Europol soit fondé sur une Convention internationale et soit mentionné à la fois dans le Traité de Maastricht et le Traité d'Amsterdam, confère à Europol une base légale claire et une plus grande légitimité qu'Interpol par exemple. De façon similaire, l'intégration de l'espace Schengen au corpus des traités développera plus avant encore l'usage du Système d'Information Schengen. Ainsi, les agences de police des Etats membres intensifieront progressivement leur coopération internationale au travers des institutions de l'UE.

11 Le développement majeur suivant et qui affectera à la fois Europol et les agences de police des Etats membres est constitué par l'amélioration de la coopération judiciaire dans les affaires criminelles. Selon le Traité, les objectifs seront définis dans des domaines tels que ceux de la facilitation et l'accélération des procédures criminelles, l'exécution des décisions et extraditions, tenter de rendre les règles de procédures criminelles compatibles, empêcher les conflits de juridiction entre les Etats, harmoniser progressivement les lois relatives au crime organisé, au terrorisme et au trafic de drogue. Compte tenu du conservatisme des hommes de loi dans les Etats membres et de la faible considération dont souffrent certains systèmes juridiques dans quelques Etats membres, le progrès vers de tels objectifs risque d'être un processus douloureusement long. Et ce d'autant plus que les répercussions dues à l'harmonisation et au rapprochement des lois sur les institutions de police ne se feront pas sentir pendant de nombreuses années. Mais la question d'une plus grande harmonie entre les 
différentes procédures criminelles et le droit pénal a été fermement inscrite à l'ordre du jour politique. La création d'Eurojust pour examiner et faciliter les solutions aux problèmes de coopération juridique en matière criminelle permet à certains d'y voir l'embryon d'un Parquet européen, de concert avec le développement d'une agence de police européenne.

12 L'intégration des règles relatives à la zone Schengen dans l'Union européenne (techniquement, il s'agit d'un domaine très problématique que d'attribuer des règles soit au premier pilier - sujet aux procédures communautaires -, soit au troisième pilier dit intergouvernemental) apporte une nouvelle base légale et une plus grande légitimité à la coopération policière dans des domaines relevant des «mesures compensatoires " considérées comme la conséquence nécessaire à la suppression des contrôles systématiques aux frontières intérieures de l'UE et au maintien des mesures communes aux frontières extérieures. Les services du SIRENE établis dans chaque Etat Schengen afin de traiter des cas d'urgence et de tester la conformité des actions avec la législation nationale font maintenant partie du système de l'Union européenne. Ceci a contraint les Etats membres à désigner un seul service de police habilité à coordonner tant les affaires de l'UE que les affaires internationales. Pour des raisons dues aux structures internes des services de police, cela s'est révélé étonnamment difficile dans certains pays (puisque la structure des services de police encourage par nature à des rivalités entre eux), mais cette pression extérieure a incité les Etats membres à désigner un tel service. Certaines agences de police, comme la gendarmerie, avec un statut militaire et qui par le passé avaient très peu de rapports avec la coopération internationale, sont aujourd'hui complètement impliquées.

L'encouragement à des opérations conjointes (décrites comme étant des actions communes par Amsterdam et Tampere) pourrait avoir des répercussions bien plus rapides et bien plus directes sur les institutions policières que toute autre avancée décrite plus haut. Ces opérations conjointes pourraient être décidées par le Conseil des Ministres ou par Europol et comprennent des activités comme la coopération opérationnelle entre les polices, les douanes et toutes autres agences chargées du respect de la loi, que ce soit pour la prévention, la recherche et l'enquête dans les affaires criminelles; la coopération dans la formation, le détachement et l'échange d'agents de liaison; l'utilisation de matériels et de données médico-légales; l'évaluation des techniques d'investigation.

14 Les agences de police sont déjà plus ou moins engagées dans ces activités. Certaines sont d'ailleurs vouées à susciter plus d'intérêt politique que d'autres. Il en est ainsi par exemple de l'encouragement à entamer une coopération dans le domaine du rassemblement, du stockage, du traitement et de l'analyse de l'information qui ont de fortes implications avec la protection des données, un sujet très sensible pour les parlementaires et les ONG. Nombre de ces activités ont fait partie du domaine informel de la coopération. L'encouragement officiel est plus ou moins bien perçu dans certains milieux policiers : utile comme argument pour obtenir des subsides, mais qui donne une publicité à des activités qui autrement sont poursuivies discrètement sans interférence politique ou administrative.

15 La caractéristique principale de la Déclaration de Tampere constitue un changement rhétorique dans un difficile équilibre entre une priorité donnée à l'application de la loi au détriment de la défense des libertés civiles. Cette ancienne priorité, évidente depuis les traités de Maastricht et d'Amsterdam, portait sur la répression du crime, la 
prévention contre l'immigration illégale et l'amélioration de la coopération entre police et justice. Le changement est intervenu avec le Traité d'Amsterdam et l'introduction d'un espace de liberté, de sécurité et de justice pour désigner ces politiques précédemment groupées sous le chapeau «Justice et Affaires intérieures ». La Déclaration de Tampere est allée encore plus loin en mettant l'accent sur la dimension positive qu'est la liberté de circulation et les mesures prises pour la faciliter. Ainsi, la défense des droits et libertés des individus, y compris celle des ressortissants des pays tiers, a été placée au centre de la Déclaration. Il s'agissait en fait d'accroître la légitimité de l'UE, qui dans tous les pays semblait souffrir d'un déclin auprès du public. L'effet direct de ce changement de priorité désormais axée sur les institutions policières risque d'être minime sauf en apportant un argument politique additionnel en faveur d'une coopération transfrontalière toujours plus étroite entre services de police.

Une proposition notable et pratique faite à Tampere a été de soumettre l'idée d'une école européenne de police, d'abord grâce à une coopération entre les différents centres de formation des polices nationales. Cette idée aura des effets à long terme sur la conception et le niveau de connaissance d'un nombre toutefois limité d'officiers de police. Mais ce qui en cascade, permettrait d'accroître le nombre d'officiers de police avertis de façon professionnelle des questions européennes. De plus, parallèlement à des mesures telles que l'action commune dans l'entraînement de la police et l'échange des savoirs, il y aurait un encouragement à l'extension d'un meilleur savoir-faire d'un Etat membre à l'autre. Ainsi, la Déclaration de Tampere envisage de créer un conseil professionnel de la police au niveau européen. Bien que les associations et syndicats de police expriment leur point de vue nuancé et que leur témoignage soit sporadiquement repris par les comités nationaux de parlementaires, un tel conseil permettrait à la police européenne d'avoir une voix, particulièrement absente jusqu'à maintenant, et d'être entendue lors des discussions politiques au sein de l'UE.

Les arguments politiques principaux à propos du système européen de coopération de police tel qu'il existe actuellement sont les suivants :

Le système est encore insuffisant, particulièrement pour ce qui relève de la fraude et des délits financiers, et le restera tant qu'un ordre juridique européen véritable dans le domaine du droit pénal ne sera pas établi. Ce système ne peut lutter contre les réalités du crime international. Le contre argument est que l'« européanisation » du droit pénal pourrait saper les bases d'un ordre juridique démocratique.

19 Le système manque de transparence car il est fondé exclusivement sur la coopération internationale. Beaucoup de développements et de nouvelles activités pourraient effectivement naître dans le secret. Ce manque de transparence est accentué par la complexité d'un système de coopération naissant. Même les experts ont parfois du mal à comprendre les implications des différents instruments de coopération: positions communes, décisions cadre, décisions et conventions. On retrouve cette difficulté lorsqu'il s'agit de comprendre les détails des accords actuels.

20 Les aménagements relatifs à la responsabilité politique et juridique sont imparfaits à cause d'un Parlement européen marginalisé et d'une Cour européenne de justice au rôle très mineur (sauf en ce qui concerne la liberté de circulation).

21 La responsabilité envers le citoyen ordinaire est virtuellement absente car rien n'est prévu pour pouvoir porter plainte auprès de l'autorité de police européenne. Toute 
plainte individuelle doit être instruite par les institutions nationales qui pourraient bien se déclarer incompétentes.

Le système de protection des informations est potentiellement déficient en raison même de la prolifération des bases de données et de la possibilité de lier et d'échanger les informations entre elles. Ainsi, l'échange d'information avec des services non européens, tout comme le système de surveillance des télécommunications entre l'UE et le FBI, constituent des menaces à l'encontre des libertés civiles.

Ces problèmes incitent certains libre-penseurs de la société civile à croire que le processus de coopération entre services de police est déjà allé trop loin et qu'une dangereuse dynamique est ainsi mise en place. Cette dynamique rend inévitable une coopération encore plus étroite entre les polices, mais sans mettre en place de véritables garde fous qui protégeraient les droits des individus et permettraient un contrôle démocratique. Le très large consensus officiel qui règne dans les Etats membres, partagé par les différentes agences de police, est qu'une coopération améliorée est essentielle pour pouvoir combattre certaines formes d'activités criminelles. Le consensus officiel prévaudra très certainement. Néanmoins, des pressions inquiètes à propos des libertés civiles, exprimées avec un certain effet dans les parlements européen et nationaux, peuvent avoir un impact sur les institutions policières et le comportement de la police.

Les institutions policières à l'échelon national ont fait partie des institutions les plus imperméables au contrôle parlementaire. Les actions légales devant les cours nationales contre des abus de la police se sont souvent révélées inefficaces et les systèmes d'instruction des plaintes contre la police font habituellement l'objet de suspicion. Ces difficultés vont certainement se répéter au niveau européen à mesure que la coopération entre les polices se développe. Mais ces difficultés n'iront pas en empirant au niveau de la coopération policière européenne, comme certains librepenseurs tendraient à le croire. La Cour européenne des droits de l'Homme a montré le chemin en condamnant une affaire de torture perpétrée par la police française et qui avait échoué devant les juridictions françaises. La Charte des droits fondamentaux de l'UE à présent en négociation pourrait bien améliorer le système de responsabilité. Le Parlement européen, en dépit de son manque de pouvoir formel, a eu beaucoup à dire dans les domaines de police et de libertés civiles. Des ONG, particulièrement Statewatch, et Justice ont apporté un courant continu d'informations à propos de ces pratiques en développement. Afin de maintenir une légitimité, les institutions européennes et les accords de coopération de police devront prendre garde aux aspects sensibles que sont la responsabilité et le respect des libertés civiles. L'européanisation des polices implique des risques mais elle offre aussi la promesse d'une plus grande sensibilité de la police elle-même à l'égard de ces domaines cruciaux. 


\section{NOTES}

1. Traduction : Nicolas Wuest-Famôse. Conférence prononcée en juin 2000 dans le cadre d'un séminaire organisé par le CERI et le Centre d'Etudes sur les Conflits.

\section{RÉSUMÉS}

The EU documents' implications for European national and European Community police agencies are explored. These agreements contribute to EU policies of liberty, justice, human rights, free movement, asylum, immigration, border control, security, and cooperation in criminal matters ; coordination in these areas will result in some integration of European police authority. Although the member states and their police agencies retain their authority over borders and policy, the European Commission has received new powers that will affect Europol's tasks and relations with national governments and police. Police roles implied by the treaty's and declaration's reforms are explained. Effects of a proposed European police academy are noted. Inadequacies of the current system of cooperation are listed ; some risks might be associated with augmenting this cooperation, but several European institutions and nongovernmental organizations effectively protect civil liberties.

\section{INDEX}

Index géographique: Union européenne

Mots-clés : construction européenne, droit européen, police

Thèmes : Traité de l'Union européenne (TUE) 